\title{
Training psychologists for the post-truth era
}

Peter Chandler

In 2016, Oxford Dictionaries selected "post-truth" as their word of the year

(https:/en.oxforddictionaries.com/word-of-the-year/word-of-the-year-2016). They describe it as an adjective defined as "related to or denoting circumstances in which objective facts are less influential in shaping public opinion than appeals to emotion or personal belief". They further note that, in this expression, "post" refers not merely to a following time period but specifically to a time period in which "the specified concept has become unimportant or irrelevant". Particularly in the context of the US Presidential election of 2016, numerous political commentators have followed the example of Steve Tesich (writing in 1992) in speculating that we are plunging inexorably into a post-truth world.

This worries a lot of people, but it needn't worry us. Psychology is, for several reasons, ideally positioned to thrive in the post-truth era. But merely to affirm this is doing the field a disservice, as it fails to acknowledge the pioneering work conducted by psychologists in helping to elucidate, exemplify and popularise the very notion of "post-truth". In this paper we survey the emergence of modern psychological methodology from the primordial soup of science and examine some of the consequences of this exciting development for our current practice. We also explore ways in which we can teach our successors to push back against some of the unwelcome encroachments on psychology from interlopers such as statisticians and philosophers of science.

\section{Green shoots of astroturf}

The behavioural sciences in general could be argued to have an atypical case of physics envy. The object of this envy is not the robustness of the mathematical basis of physics, but the sheer convenience of being able to conduct an experimental science based on the behaviour of matter which is abundant, freely interchangeable, and not required to give informed consent, even when being annihilated in a particle accelerator.

An impractical solution to this problem is to enhance statistical methods to take into account aspects of the unwanted variability in behavioral experiments: for instance, using mixed models to address the fact that participants are effectively (pseudo-)randomly sampled from a population of (pseudo-)typical individuals. Unfortunately the advances in computational power that have made such methods tractable have not been paralleled by the kinds of advances in comprehension power that would enable researchers to understand the implications of the outputs.

A more practical solution, already widely adopted before such computational advances were made, is simply to routinize the process of creating academic outputs. A crucial step in this direction was adopting the Neyman-Pearson approach to the use of p-values: that is, taking the view that a critical value for $\alpha$ should be determined in advance. The choice of $\alpha$ could be said to parameterise a whole field of experimental enquiry: adopting a very small $\alpha$-value as the standard for a field would result in research progress that was at best glacial, involving the publication of a small number of "highquality" findings. Adopting a large $\alpha$-value, such as 0.05 , would naturally result in a dynamic, exciting field with excellent publication prospects. The publication of masses of non-replicable findings would be a consequence of this approach, but not an undesirable one, given that each incorrect finding published opens up not only the possibility of rebutting it in a separate paper but also the possibility of building further incorrect but publishable findings upon it. 


\section{Publishing in the face of reality}

Taking a generous $\alpha=0.05$ as the criterion for statistical significance, and hence the rejection of null hypotheses and the adoption of alternatives, has predictable consequences for the design of experiments. The most efficient way towards publication, and hence career advancement, involves conducting numerous small experiments (Bakker et al., 2012). As critics of the "file-drawer problem" in psychology have noted, one in twenty attempts to detect an effect that does not exist will demonstrate (to the satisfaction of the statistical method, in that given instance) that in fact it does exist. But the smart experimenter should not have to rely on repetition ad nauseam of small experiments in the hope of locating false positives, any more than she should have to depend on outright falsification of data. On the contrary, as long as the experimental materials are set up to yield evidence of a difference, the experimenter can obtain significance straightforwardly.

What I mean is this. In the idealised view, one devises a theory, identifies a distinctive prediction made by that theory, and tests whether that prediction is borne out: for instance, whether a given manipulation leads to higher decision accuracy, faster reaction times, a larger N400 response, or what have you. In practice, many different factors bear upon the outcome, including not only a lot of things we understand but a lot of things that haven't yet been theorised about. So we might compare the reading times for two word lists, to see whether - let's say - their consistency of CV letter order makes a difference. Controlling for everything known to be relevant, we might yet have an intuition, prior to running the experiment, that one list is easier than another. If not, we can keep adjusting the lists until we do. If the intuition runs in the direction that our theory predicts, we run the experiment. (If not, we can either change the lists or change our theory, according to taste.)

Does this count as a violation of statistical best practice? Perhaps in terms of the spirit of the law, but not the letter. Indeed, if we do consider it a violation, the entire discipline of psycholinguistics is hanging by a thread: a major theme in the linguistics sales pitch is that we all have access to a rich set of intuitions about language. It would hardly be surprising if a skilled user of language could inspect two lists of words and form a reliable opinion about which of them was likely to be read more quickly, even in the absence of any knowledge about what the intended manipulation was. (Perhaps an experienced neurolinguist will also have intuitions about which will yield larger N400s.) If we do have such knowledge (and experimenters have helpfully refrained from finding out whether this is actually the case), we can hardly be expected to check it at the door of the lab when we go in to design our experiments.

In practice, we needn't go so far to achieve the effect that we desire. We can process hypotheses very rapidly: we come up with a manipulation to test, construct some items that would populate the two test lists, and then look at them and introspect about whether the experiment that we would run with those test lists would disclose the desired effect. If not, we try a different manipulation. In this way, it may be that we can weed out most of the null effects just by inspection, and without populating our file drawer with any real data whatsoever.

By certain standards this kind of practice would be regarded as lacking in scientific integrity. Richard Feynman famously remarked that science required its practitioners to eliminate all reasonable alternative explanations for their data. His characterisation of this as a kind of "leaning over backwards" would immediately be understood by any osteopath as a satirical injunction against taking this notion of integrity too seriously.

Indeed, Feynman made numerous pertinent remarks on the philosophy of science, many of which have been misinterpreted by the practitioners of "hard sciences", while engaging in their favored pastime of looking for sticks with which to beat the humanities. His celebrated remarks on "cargo cult science" are a case in point. Feynman compared the propensity of psychologists to adopt the trappings of other empirical scientists to the actions of Pacific islanders who built fake airstrips after the Second 
World War in the hope of attracting planes. His mistake is to see their actions purely in terms of the teleological purpose that he attaches to them - that is, causing planes to land - by which standard they are obviously deficient. But the actions of the cargo cultists are wholly defensible if we think of their actions as being intended to create something that looks like an airstrip, as a talisman - or, more practically, to procrastinate on more functional but less culturally valuable tasks such as fishing or the production of copra. By analogy, the criticism of psychology amounts to an attack on its inability reliably to produce a certain kind of knowledge (specifically, a body of reproducible facts corresponding to the situation in the world), and a failure to appreciate its core aims as an intellectual activity.

In the same spirit, Feynman argues that "the first principle is that you must not fool yourself - and you are the easiest person to fool". This is certainly good advice, but its relevance to practice is far greater in the hard sciences. I take it as uncontroversial that to "fool yourself" means to be led to a false belief. The simplest way to obviate that in the domain of psychology appears simply to be not to believe one's own findings. While Feynman argues that, after having not fooled oneself, not fooling other people is simply a matter of honesty, I would argue that the causal chain goes the other way for us. After not having believed anyone else's findings, not believing one's own is a sensible generalisation. Once again, I must stress that this point does not go to the validity of psychology as an academic discipline, merely to its status as a particular kind of academic discipline (specifically, the kind whose practitioners make snarky remarks about other disciplines).

\section{Maintaining a diverse intellectual ecology}

The spirit of the criticisms discussed in the preceding section is redolent of the rather vague nexus of objections to the status quo evoked by the phrase "drain the swamp", another commonplace of recent political discourse. This phrase dates back at least to 1903, when Winfield R. Gaylord of the Social Democratic Party observed that "Socialists are not satisfied with killing a few of the mosquitoes which come from the capitalist swamp; they want to drain the swamp".

Thus, one striking point about the political use of the phrase is that it has been variously used to attack a diverse range of political positions and groups: capitalists (Gaylord), 'Big Government' (Reagan), terrorists (Pelosi), lobbyists (Trump), etc. Its function, in sociological terms, is to inscribe an othering discourse. And if this means anything (a point which sociology is still debating), it means the process of installing some identified set of individuals as an out-group, an aim which has been all too evident in the attitude of 'hard' scientists towards researchers in psychology. The goal of the likes of Feynman is, in its own terms, perhaps even admirable in its intellectual self-confidence: they wish to prevent psychology from incubating attitudes that they deem injurious to the scientific body politic.

It is crucial to the original resonance of the phrase "drain the swamp" that swamps are traditionally regarded as breeding-grounds for the Anopheles mosquito which is the vector for malaria. However, swamps are not solely the preserve of mosquitoes, but are in many cases havens of biodiversity; and in any case, malaria was eradicated in the US in the early 1950s, according to the Centers for Disease Control and Prevention. Thus, given the current ecological state of play, any endeavors to drain swamps are liable to incur environmental losses while bringing about relatively tenuous, if even extant, public health benefits.

The parallel I wish to draw here is, I hope, obvious. The idea of 'draining the swamp' of social psychology in order to eliminate practices that some scientists find objectionable is palpably unnecessary given current societal conditions. The potential damage to society arising from current practices in psychology is mitigated by a host of factors, including those mentioned earlier in this paper. To the extent that social psychology generates findings, these findings are not considered to be necessarily indicative of external reality. They are, to a large extent, not trusted by the public, either because of widespread skepticism about the nature of intellectual enquiry in general, specific 
collective doubts about the plausibility of particular published claims, or most simply because the results are never made available to public gaze. Thus, the putative problems largely fail to arise, and could indeed be seen as a strawman which is being attacked as part of a broader ideological agenda that aims to assimilate all fields of intellectual enquiry to the hard sciences. Such an attack should be resisted on the grounds of intellectual and methodological pluralism and multiculturalism, which are widely agreed to be highly positively valent words.

\section{The Seven Inspiring Virtues of Psychology}

A particular challenge at this point in time is to maintain morale in a discipline beset by such vehement internally- and externally-generated criticism. Rather than further depress ourselves with the likes of Chambers's (2017) "The Seven Deadly Sins of Psychology”, we should draw succour from the many strengths that the discipline already possesses, and focus on how we can ensure that psychology in the future will be a huge, big, beautiful subject stretching from coast to coast.

\section{The Virtue of Positivity}

One of the most exciting aspects of experimental psychology is the field's success in obtaining positive findings. Popular and successful results in the field are almost invariably of this kind: it would, for example, be newsworthy that meat-eating causes selfishness

(http://www.dutchdailynews.com/meat-eaters-selfish-less-social/), whereas a demonstration that this had no effect would be unlikely to attract much attention.

Nature has provided our field with something of a head-start - it would hardly be surprising if, for example, every measurable aspect of personality was correlated in some way with the outcome of any given psychological test. However, being endowed with this kind of bounty is not always an advantage, and we are in constant danger of succumbing to what economists (rather serendipitously) call the Dutch disease, where growth in one sector is causally linked to decline in other sectors. That is to say, it would be easy to give in and focus entirely on the demonstration of actual facts, given how many there are to be tracked down, and lose focus on the more challenging and instructive business of providing convincing proofs of claims that are not actually true.

Worse still is the emerging trend within which researchers actually attempt to rebut or 'debunk' socalled 'false' claims. Accepting the point that absence of evidence is not evidence of absence, the upshot of most such studies is simply to place on the record our collective ignorance as to whether a claim is true or not, which can hardly be regarded as the pinnacle of scientific accomplishment by anyone's standards.

Nevertheless, the publication practices in our field clearly set us upon the right path. Positive results are publishable; negative results are not. Positive results can only be satisfactorily countermanded by more interesting positive results. The advantages to this approach are seen not only in the rapid growth of publications on a given topic, but also in the rapid development of theory, which can be seen to chase the ever-burgeoning pack of experimental findings. It is perhaps not too fanciful to see this as a recapitulation of some of the most inspiring principles of Bahá'u'lláh, in that we can consider each result to be true for its time and to guide us on the path to ultimate enlightenment, even though the sum total of the literature may give the appearance of self-contradiction.

\section{The Virtue of Flexibility}

For any properly-conducted experiment, clearly we cannot guarantee knowing in advance whether the hypotheses motivating the work are likely to be borne out by the study itself. We can rely on useful heuristics - for instance, assuming that our hypotheses are false at some sufficiently fine level of detail, and assuming that we can always demonstrate $\mathrm{p}<0.05$ given a large enough sample - but we cannot be sure that a practicably small experiment is going to speak to the initial topics of interest. 
Fortunately, because we are engaged in a behavioral science, this need not matter. When we conduct an experiment with human participants we are inarguably collecting data concerning the behavior of those participants. It is hardly conceivable that the resulting data will have no points of interest whatsoever, or that no interesting null hypothesis could be rejected because of these data (Simmons, Nelson, \& Simonsohn, 2011). The challenge, risen to on a daily basis by practitioners across the field, is to retrofit the past experiment with sufficient points of theoretical interest to justify the work that has been put in to conducting it (cf. Bem, 2003).

Such an endeavour is not merely a matter of ego-boosting self-justification: it could reasonably be said to be a moral imperative. Participants volunteer their time on the understanding that they are performing a service to the field (a possible exception being neuroimaging studies, which might be more accurately categorised as participants gambling with their health for money). If the data garnered from the study are not of theoretical interest, then participants have been misled, to an extent that is morally if not legally worrisome. Once again, on the understanding that participants expect their work to lead to progress in psychology, any lack of real-world relevance exhibited by the resulting scientific outputs does not pose a corresponding moral problem - as argued earlier, progress in psychology is not reducible to the discovery of true facts about reality.

This kind of theoretical flexibility can be supported by appeal to distinct categories of intellectual practice elsewhere, ranging from fine art to hard science. It is absurd to imagine that when Cézanne sat down to paint the view of Mont Sainte-Victoire that he had a preconceived notion of precisely what effects of light and shade would be most interesting on a given occasion. It is a matter of public record that institutions such as CERN conduct experiments in high-energy physics with a view to establishing whether or not new kinds of particle can be detected, with the focus turning to specific discoveries only when there is a strong expectation that such discoveries have been made. Given that CERN receives a budget in excess of CHF 1 billion per year, and for that matter that Cézanne's record sale price exceeds $\$ 250$ million, flexibility offers practical as well as spiritual rewards.

\section{The Virtue of Generosity}

The spirit of the Golden Rule - "do unto others as you would have them do unto you" - is a cornerstone of academic practice, albeit one that has been undermined by certain regrettable developments in connection with the so-called "replication crisis" (Gilbert et al. 2016). Until recently, a high-impact publication in an estimable journal was nothing but a cause for admiration, especially if backed up with friendly media attention. Now, such events are received coolly, or even with bitterness, by the academic community at large (for a particularly salient example of this "bad loser" mentality, see e.g. http://andrewgelman.com/2017/03/28/association-psychological-pseudoscience/).

It would be uncharitable to assume that researchers who raise the issue of replicability are motivated by professional jealousy, rather than simply by a misunderstanding of the nature of experimental psychology. That said, we are not running a charity here. Professional jealousy of this kind clouds the judgment, to the extent that people allow themselves to become obsessed with the question of whether the academic record is "heavily polluted" with "false" findings. Given that failing to replicate a result does not prove its falsity, and that the resources to put together such a proof are typically not available to the researcher, it is hard to escape the conclusions that this cabal of psychologists (described by some of our more hot-blooded colleagues as "methodological terrorists") are tilting at windmills.

A more positive and magnanimous approach benefits the field and perhaps the mental health of the individual. If some rival is better at churning out publishable findings, we must tolerate that, and if we require professional help to do so, it should be provided by a therapist rather than a statistician. Of course the findings won't replicate: it is unreasonable and ideologically narrow of us to expect or require this. Psychology is not like engineering, or medicine, where there are consequences if things don't work. There's nothing that needs to "work" - our job is to create compelling stories about the 
inner life of humanity. Exhibiting counterintuitive findings not only makes us look good but makes our readers feel enlightened. The more precise the findings can be made, the more enlightening they are. As a colleague at the University of Chicago once sagely observed, "We scientists do not have to test predictions, our job is to say things that sound intelligent". It is by applying the scientific method that we can generate such things to say, and it is through careful education in quantitative methods that we can ensure our findings are richer and more rewarding for the reader than the arrant qualitative speculations of politicians, journalists and religious leaders.

\section{The Virtue of Discretion}

The process of turning data into findings is a complex one. Confronted with full evidence of this complexity, a casual onlooker might wonder whether this complexity is warranted - indeed, they might form the opinion that the process actually should be a lot simpler. This is an extremely dangerous point of view, and it should not be necessary to elect such an onlooker to the US Presidency in order to convince the reader of this point.

The important point to hold in mind is (to paraphrase John Godfrey Saxe) that scientific discoveries, like sausages, cease to inspire respect in proportion as we know how they are made. Is it possible to read of the discovery of penicillin without feeling a simmering resentment for the plaudits earned by Fleming as a consequence of his carelessness (and notwithstanding his inability to take full advantage of it)?

Traditionally, researchers in psychology have well understood the advantages of discretion about the precise nature of the underlying data, materials, lab conditions, participant profiles, and lab members' track records of academic malpractice. The full disclosure of such information can only fuel unjustified suspicion about the value of findings (as well as much irrelevant speculation about their "veracity" or "replicability", if the reader will forgive my return to this side-track).

Now, increasingly, pressure is being brought to bear to make such information publicly accessible. This is clearly in the interests of researchers who lack lab resources and whose publication prospects depend upon free-riding in the form of reanalysis or meta-analysis of published findings. But encouraging such behavior not only fosters uncollegiality; it also results in a lack of clarity in the public mind about the status of scientific findings, which is extremely detrimental both to psychology and to fields primarily interested in external validity.

The important point to stress is that the banquet that is the published psychological literature does not need to be disrupted by unwelcome guests asking about the recipe - whether it's because they admire the hors d'oeuvres or because they are having a life-threatening allergic reaction to the entrée. The key word must be 'omakase' - loosely speaking, 'trust the chef'.

\section{The Virtue of Open-Mindedness}

As alluded to earlier, much of the excitement of experimental work comes from not knowing what one might find. In the case of psychology, there is scarcely any limit to what we might be able to discover through our agreed procedures for scientific enquiry: at least, it is hard to see where such a limit would come from, in that reality itself need not figure in the calculation.

Unexpected results call for a particular kind of appreciation. A regrettable recent trend is to greet them with denial - people object vehemently to the very notion that, for example, lonely people might take longer showers (Bargh \& Shalev, 2012), or speakers of languages that lack future tense marking might save more for a rainy day (Chen, 2013), and so on. Again, this is an unhealthy attitude. It appears to arise because researchers - who should know better - insist on evaluating other people's findings as though they are to be treated as claims about reality, instead of as the outputs of a 
legitimate process for generating research findings. This is a little like objecting to a Damien Hirst spin-painting on the basis that it doesn't resemble a picture of any known artefact.

Indeed, even if we insist on construing the findings as claims about reality, the vehemence of the objections is hard to defend. In reality, either it's true that lonely people take longer showers or it's not true. If it's true then objecting to its factuality is perverse; if it's not true then objecting to its factuality is unnecessary.

But if our aim is to develop a full and proper appreciation of psychology as a discipline, these claims about truth or falsity remain a sideshow. We can, and usually do, appreciate research findings as part of the rich tapestry of human experience - indeed, as part of the way we make sense of the world. Dismissing them on ideological grounds, or because they fail to adhere to known principles, diminishes us all by attempting to circumscribe the sum total of admissible human culture on highhanded scientistic grounds.

\section{The Virtue of Respectability}

Although in the previous section I argued for the status of psychological research as a part of human culture, I would not wish to defend a postmodern approach to that culture to the extent that all contributions are deemed equally valid. Indeed, the whole purpose of training in experimental psychology is to ensure that its practitioners are able to adhere to the cultural norms of the programme. I take it as essential that we strive to produce work that embodies the essence of this intellectual enterprise. If we disregard the methods, we will produce findings that may or may not correspond to reality (indeed, may be systematically more or less likely to correspond to reality), but importantly cannot truly be regarded as psychological research.

An important part of the process of psychological enquiry is, therefore, gaining the imprimatur of a well-regarded journal. Simply to publish our work in an unregulated online repository, or indeed in a lightly-reviewed pay-to-play journal, represents a deviation from this practice. More importantly, it engenders confusion in the mind of the reader as to whether the work is or is not to be considered canonical.

The crucial step of peer review cannot be done away with lightly. The existing system of peer review, as implemented by most respectable journals, has several important points in its favor. Firstly, it enforces adherence to existing methods and discourages wanton innovation, for instance through the introduction of new analytic techniques that are not widely understood. Such innovations place a burden on other practitioners in the field and could be considered uncollegial for this reason. Secondly, it is not so intrusive that it blocks researchers from engaging in the kind of flexibility discussed earlier on: pre-registration is not normally required, so the system agreeably accommodates post hoc changes to the experimental hypotheses, statistical analyses, and so on. Thirdly, it reinforces power relations within the field, which are essential to the maintenance of a well-ordered discipline, and has proved somewhat effective in preventing the kind of schisms that have characterised fields as similar as Christianity, Chomskyan linguistics, and heavyweight boxing.

\section{The Virtue of Objectivity}

Finally, psychology has been exemplary in its embrace of objective methods for measuring the quality of our work. Our impression of our colleagues' ability can hardly be used as a yardstick for hiring and tenure decisions, colored as it so often is by ad hominem considerations such as agreeableness, conscientiousness, extraversion, neuroticism, and personal hygiene. Rather, we have access to quantitative data: impact factors, citation counts, the receipt of grant funding, and so on. These represent a vital and independent measure of a researcher's capabilities, which is essential when we are called upon to evaluate that individual in some way: deciding whether to accept their paper for publication, whether to cite their work in our latest paper, whether to favor their grant application, and 
so on. Without such measures - and even now, there are those who would try to eliminate them from consideration - we would be forced into nothing other than an endless self-perpetuating loop.

\section{Conclusion}

Psychology, and specifically social psychology, has the potential to be the most inspiring of all the many fields of intellectual enquiry. But in order to realise this potential, we must continue to train our young researchers in the established ways, and resist those external forces that pressure us to lower our standards and focus on the uncreative pursuit of actual knowledge. Such developments force us into a "race to the bottom" with activities such as physics, engineering and medicine.

I envisage a future of beautiful psychology in which an army of researchers - more people than ever before - take the helm of psychology and boldly find what no-one has found before, independently of whether anyone will be able to find it again.

In conclusion, I invite the reader to ponder on the following story, drawn from my personal experience. Recently I was in a mall, and saw a customer who was considering buying a set of bathroom scales. She complained to the clerk that the scales she was trying gave a wildly different outcome every time she stepped on them. The clerk wisely explained that the scales are not meant to be used consecutively in that way, because one only needs one number to describe one's weight, not a set of different numbers. It is sufficient to step on the scales once, take the measurement, and then get on with one's life. In that spirit, instead of preregistering, sharing data and mindlessly attempting to replicate the findings of others, we should go out and create new knowledge, epic truths that amuse, amaze and alarm us. 


\section{REFERENCES}

Bakker, M., van Dijk, A., \& Wicherts, J. M. (2012). The rules of the game called psychological science. Perspectives on Psychological Science, 7(6), 543-554.

Bargh, J. A., \& Shalev, I. (2012). The substitutability of physical and social warmth in daily life. Emotion, 12(1), 154.

Bem, D. (2003). J.,(2003) Writing the Empirical Journal Article. Darley, J., M., Yanna, M., P., and Roediger III, H., L., (Eds).

Chambers, C. (2017). The Seven Deadly Sins of Psychology: A Manifesto for Reforming the Culture of Scientific Practice: Princeton University Press.

Chen, M. K. (2013). The effect of language on economic behavior: Evidence from savings rates, health behaviors, and retirement assets. The American Economic Review, 103(2), 690731.

Gilbert, D., King, G., Pettigrew, S., \& Wilson, T. (2016). More on "Estimating the Reproducibility of Psychological Science". Available at projects.iq.harvard. edu/files/psychologyreplications/files/gkpw_post_publication_response. pdf.

Simmons, J. P., Nelson, L. D., \& Simonsohn, U. (2011). False-positive psychology: Undisclosed flexibility in data collection and analysis allows presenting anything as significant. Psychological science, 22(11), 1359-1366. 